\title{
A diabatization protocol that includes spin-orbit coupling
}

\author{
Tao Zeng \\ Department of Chemistry, Carleton University, Ottawa, Ontario K1S5B6, Canada
}

\begin{abstract}
A diabatization protocol that combines the recently developed model space diabatization scheme and a mean-field treatment for spin-orbit coupling is presented. The protocol is highly efficient and easy to use. Its robustness is demonstrated in the calculations of the diabatic spin-orbit matrix elements of $\mathrm{PH}_{3}^{+}, \mathrm{AsH}_{3}^{+}$, and $\mathrm{SbH}_{3}^{+}$. It captures subtle geometry-dependence of the spin-orbit couplings and provides wave function information to explain the origin of the dependence.
\end{abstract}




\section{INTRODUCTION}

In quantum chemistry and molecular physics, adiabatic states (adiabats) are the eigenstates of the electronic Hamiltonian operator. They may vary rapidly, especially when they are close in energy, leading to the non-adiabatic couplings (NACs) and invalidating the BornOppenheimer (BO) approximation. ${ }^{1,2}$ The NACs are represented by the derivative coupling matrix element $\left\langle\Psi_{I}|\nabla| \Psi_{J}\right\rangle$, where $\{\Psi\}$ denote the adiabats and $\nabla$ the vector derivative of nuclear coordinates. The NACs obliterate the classical dynamics picture of nuclear motion on a single potential energy surface (PES); in non-adiabatic dynamics, nuclear wave packet evolves on several PESs and switch among the surfaces through internal conversion. ${ }^{3}$ Wherever the adiabats become degenerate and form a conical intersection, the NACs become singular and the adiabatic PESs become cuspidal. ${ }^{2}$ An avenue to avoid the complication of the singularity and the cuspidal PESs is to switch to the diabatic picture. ${ }^{4}$ (Quasi-)diabatic states (diabats, denoted by $\{\chi\}$ ) maintain their characters along the molecule's structural change, so that their NAC elements $\left\langle\chi_{I}|\nabla| \chi_{J}\right\rangle \approx 0 .{ }^{5-9}$ A group of adiabats that feature substantial NACs within themselves can be diabatized to the same number of diabats. The approximation that ignores the NACs between a group of states and the rest is called the group (or generalized) Born-Oppenheimer approximation (GBOA). ${ }^{1,9}\{\chi\}$ are not eigenstates of the electronic Hamiltonian $\hat{H}_{e l}$; the couplings between electronic states are represented by the off-diagonal matrix elements $\left\langle\chi_{I}\left|\hat{H}_{e l}\right| \chi_{J}\right\rangle$. In the representation of the slowly varying $\{\chi\}$, the $\left\langle\chi_{I}\left|\hat{H}_{e l}\right| \chi_{J}\right\rangle$ elements, diagonal or not, are smooth functions of the nuclear degrees of freedom.

Diabatization methods can be largely classified as property-based, ${ }^{10-15}$ by ansatz, ${ }^{7,16-19}$ and wave-function-based. ${ }^{20-30}$ The property-based methods construct diabats based on their differences in properties. Diabatizations by ansatz expand the $\left\langle\chi_{I}\left|\hat{H}_{e l}\right| \chi_{J}\right\rangle$ matrix elements around a conical intersection. The expansion is parametrized through fitting the eigenvalues of the matrix against the adiabatic energies. Wave-function-based methods either calculate the $\left\langle\Psi_{I}|\nabla| \Psi_{J}\right\rangle$ elements and then transform the states to minimize them, or transform the adiabats to character-preserving diabats so that implicitly, $\left\langle\chi_{I}|\nabla| \chi_{J}\right\rangle \approx 0$. Recently, a hybrid diabatization scheme that employs both wave function information and by-ansatz model has been proposed. ${ }^{31}$

Spin-orbit (SO) coupling (SOC) is another important mechanism that couples different 
electronic states. ${ }^{32-36}$ It can couple states of different spin multiplicities, leading to intersystem crossings (ISCs) in dynamics and spin-forbidden transitions in spectroscopy. Considering both the one- and two-electron SO interactions, the magnitude of SOC increases approximately as the square of nuclear charge. ${ }^{37-39}$ Spatially degenerate electronic states with $S \neq 0$ ( $S$, the total spin quantum number), such as the ${ }^{2} E$ states of molecules with trigonal symmetry and the ${ }^{3} \mathrm{~T}$ states of molecules with tetrahedral symmetry, are subject to the more pronounced, first order SOC. ${ }^{33}$

Certainly, both the NAC and the SOC play significant roles in vibronic spectroscopy and excited states dynamics. One example that involves both types of coupling is the ISC of psoralen: ${ }^{40}$ considering only SOC gives a $<10^{5} \mathrm{~s}^{-1}$ rate constant for the transition from the first singlet to the first triplet state; when considering vibronic SOC, the rate constant is increased to $3 \times 10^{8} \mathrm{~s}^{-1}$. As a conventional simplification, SOC is assumed to be independent of nuclear structure. ${ }^{41-45}$ While this approximation works well for many cases, it is not universally applicable. Domcke and coworkers pioneered in investigating the geometrydependence of SOC and found intricate interplay between the Jahn-Teller (JT) effect and SOC. ${ }^{46-50}$ Especially, the JT effect can arise solely from SOC. These findings necessitate a diabatization scheme that can describe the geometry-dependence of SOC. There have been attempts to include SOC in diabatization: (1) diabatization by ansatz. ${ }^{51-53}$ The procedure is similar to the non-SOC counterpart introduced above, but with SOC incorporated in the adiabatic PESs. It works well when SOC is of large magnitude, especially larger than the fitting error of the diabatization; (2) projection of atomic SOC. SOC in a molecule can be viewed as a summation of individual contributions from constituent atoms. One can express molecular diabats using wave functions of the constituent atoms, and then project the atomic SOCs to the diabats. The recently developed Effective Relativistic Coupling by Asymptotic Representation (ERCAR) diabatization of Eisfeld and coworkers ${ }^{54-56}$ is based on this strategy; (3) a straightforward way is to calculate SOCs of adiabats, and then convert them to the representation of diabats. ${ }^{57,58}$ This type of methods require accurate evaluation of molecular SOC and a robust diabatization scheme for the non-SOC states.

In this work, we propose an SOC-diabatization protocol that belongs to the third type of methods. The protocol combines the recently developed model space diabatization (MSD) ${ }^{28}$ for non-SOC states and an SOC treatment that includes the mean-field 2-electron interaction. In Section II, we outline the protocol. $\mathrm{PH}_{3}^{+}, \mathrm{AsH}_{3}^{+}$, and $\mathrm{SbH}_{3}^{+}$are then used as 
examples to demonstrate the validity and advantages of the protocol in Section III. Section IV concludes the paper.

\section{METHODOLOGY}

\section{A. Model Space Diabatization snd Spin-Orbit Coupling Calculation}

The details of the model space diabatization (MSD) scheme of Li et al. are given in Ref. 28. In brief, this method is rooted in Atchity and Ruedenberg's idea of configuration uniformity: ${ }^{23,24}$ adiabats are rotated to have maximum projections onto the respective spaces of diabatic prototypes. Here, we only introduce our implementation of this method. We have adapted MSD to the general multiconfigurational quasidegenerate perturbation theory (GMC-QDPT) calculations, ${ }^{59-61}$ which allow different types of active space, e.g., complete active space (CAS), restricted active space (RAS), occupation restricted multiple active space (ORMAS), ${ }^{62}$ etc. This adaptation significantly enhances the applicability of MSD. The diabatization requires to express the adiabats in a set of diabatic molecular orbitals (DMOs) that maintain their character along nuclear distortion. In this paper, DMOs are obtained through rotating the canonical molecular orbitals (CMOs) to have maximum overlaps with some reference orbitals at reference structures. Atchity and Ruedenberg's scheme ${ }^{63}$ is employed to transform the expansion coefficients of Slater determinants in adiabats when expressing the adiabats in DMOs. Using this transformation scheme, we can easily fix the phases of the diabats. The extra steps proposed in Section II.C.3 in Ref. 28 to keep consistent phases for diabats can be skipped. This is another technical improvement that promotes the usage of MSD. The diabatization gives the diabats that are a unitary transformation of the adiabats:

$$
\left|\chi_{I}\right\rangle=\sum_{J}\left|\Psi_{J}\right\rangle U_{J I}
$$

The robustness of this implementation of MSD is demonstrated in our recent studies of singlet fission, ${ }^{64,65}$ in which diabatization for up to 13 states was successfully carried out.

We ask the readers to refer to the excellent review articles ${ }^{32-34,36}$ for discussion of the SOC and its effects in chemistry. Here, we only briefly introduce how we treat this interaction in our diabatization protocol. We employ a mean-field treatment similar to that of Hess et al. ${ }^{66}$ to calculate the SOC matrix elements between adiabats, $\left\langle\Psi_{I}\left|\hat{H}_{S O}\right| \Psi_{J}\right\rangle$. While the 
one-electron SOC is directly calculated following the Slater-Condon rule, the more timeconsuming two-electron SOC is approximated as an effective screening of the one-electron interaction. Technical details of our SOC calculation are given in Section S.I in the supporting information (SI), along with a comparison between our calculated atomic SO splittings for $p$-block elements and experimental values. The comparison demonstrates the accuracy of our SOC calculation.

Once the $U$ matrix in Eq. 1 and the $\left\langle\Psi_{I}\left|\hat{H}_{S O}\right| \Psi_{J}\right\rangle$ matrix are calculated, the SOCs between diabats are obtained through the transformation

$$
\left\langle\chi_{I}\left|\hat{H}_{S O}\right| \chi_{J}\right\rangle=\sum_{K L} U_{K I}\left\langle\Psi_{K}\left|\hat{H}_{S O}\right| \Psi_{L}\right\rangle U_{L J}
$$

With such a direct calculation of the diabatic SO matrix elements, there is no need for the a priori knowledge of the SOCs of the constituent atoms and the decomposition of the diabatic wave functions into atomic contributions. In general, the diabatization and SOC evaluation together add only a small fraction of compute time for the GMC-QDPT single point calculation. For instance, for the most time-consuming $\mathrm{SbH}_{3}^{+}$calculations in this work, about $20 \%$ more time is needed for the diabatization and SOC steps. The two steps are never the bottlenecks of a calculation; the efficiency of the protocol is promising.

\section{B. Computational Details}

All single point energies, wave functions, diabatic couplings, and SO matrix elements are prepared with GMC-QDPT calculations using Granovsky's zeroth order Hamiltonian ${ }^{67}$ and the $0.02 E_{H}^{2}$ intruder state avoidance parameter. ${ }^{68,69}$ The 7 orbitals and 7 electrons (7o7e) full valence active space is used for the pnicogen hydride cations. The structures of the pnicogen hydrides are optimized using density functional theory with the B3LYP functional. ${ }^{70-73}$ The cc-pVTZ basis set ${ }^{74-76}$ are used for $\mathrm{PH}_{3}$ and $\mathrm{AsH}_{3}$ and their cations. For the heavier $\mathrm{SbH}_{3}$ and its cation, the Sapporo-DK-TZP ${ }^{77}$ basis set is used, along with the third order Douglas-Kroll (DK3) relativistic Hamiltonian. ${ }^{78-81}$ All calculations were carried out using the GAMESS-US program package. ${ }^{82,83}$ The SOC-diabatization protocol has been implemented into a development version of the program. 


\section{PNICOGEN CATIONS AS EXAMPLES}

A good SOC-diabatization scheme should produce diabatic SO matrix elements that vary smoothly with respect to nuclear distortion, and the variation should be physically sound. To validate the protocol, we use it to investigate the geometry-dependence of diabatic SOCs for $\mathrm{PH}_{3}^{+}, \mathrm{AsH}_{3}^{+}$, and $\mathrm{SbH}_{3}^{+}$. These cations feature both vibronic coupling and SOC. Their trigonal symmetry allows for the E-type spatial degeneracy, and hence the JT effect. ${ }^{84}$ The interaction between the $E$ and an $A_{1}$ state also gives the pseudo-JT (pJT) effect. ${ }^{84}$ The spatial degeneracy delivers electronic orbital angular momentum, which interacts with the doublet spin and gives SOC. Constructing SOC-vibronic models for these cations is challenging. The light mass of $\mathrm{H}$ atoms and the small force constants of the $\mathrm{H}-\mathrm{A}-\mathrm{H}$ (A for a pnicogen atom) bending modes allows for large amplitude, anharmonic motions along these JT- and pJT-active modes. ${ }^{85,86}$ The description of the diabatic Hamiltonian matrices of the cations requires high order expansions in nuclear coordinates (e.g., up to the 8-th order for $\left.\mathrm{PH}_{3}^{+}\right) .{ }^{87,88}$ It is reasonable to expect the SOCs of the cations to display substantial geometry-dependence over the large range of nuclear motion.

With more fitting parameters, the high-order expansion inevitably complicates the diabatization by ansatz. The $\left\langle\chi_{I}\left|\hat{H}_{S O}\right| \chi_{J}\right\rangle$ elements of small magnitude are more subject to this complication. For instance, the $\left\langle\chi_{I}\left|\hat{H}_{S O}\right| \chi_{J}\right\rangle$ elements of $\mathrm{PH}_{3}^{+}$have magnitudes of tens of $\mathrm{cm}^{-1}$ (see Section III.A.1 below) and are easily blurred in the $\sim 0.05 \mathrm{eV}\left(400 \mathrm{~cm}^{-1}\right)$ fitting error ${ }^{88}$ of the diabatization by ansatz. In the present wave-function-based protocol, those matrix elements are directly calculated. The fitting errors only enter the analytical representations of the matrix elements, not the matrix elements themselves (see more discussion in the end of Section III.C). This is an advantage of using the present protocol. It has been revealed in Ref. 88 that the $e$ bending modes of $\mathrm{PH}_{3}^{+}$are the most JT- and pJTactive in coupling the three lowest electronic states (belonging to the $E$ and $A_{1}$ irreducible representations (irreps) of the $C_{3 v}$ symmetry). We hence focus on the variation of the SO elements along the $e$ bending of the cations. The convention of using upper case symbols for electronic state irreps and lower case symbols for vibrational and orbital irreps is followed. 


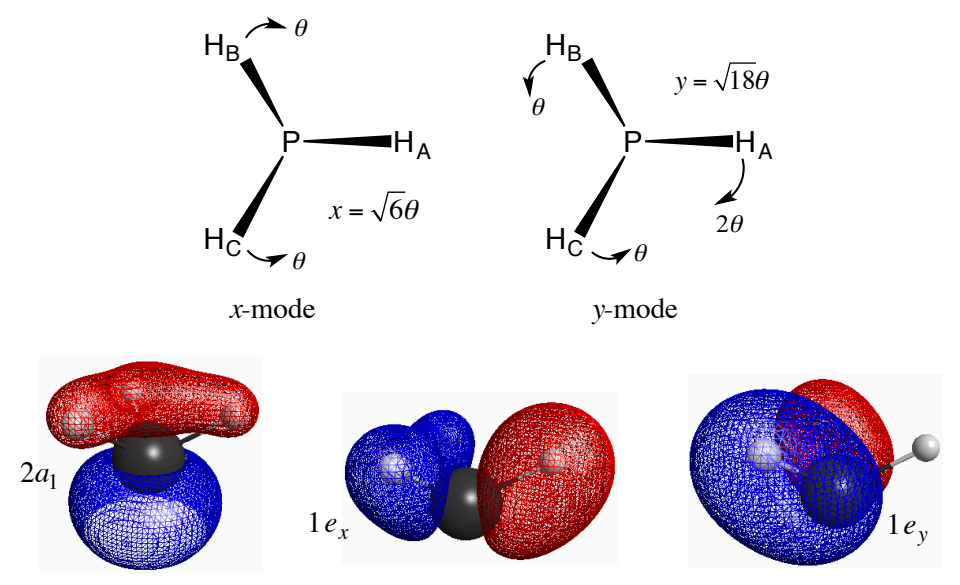

FIG. 1. The bending modes and the orbitals from which an electron is removed to give, from left to right, the $Z, X$, and $Y$ states of $\mathrm{PH}_{3}^{+}$.

\section{A. Phosphine cation}

$\mathrm{PH}_{3}^{+}$has the lightest central atom among the cations considered and hence the smallest SOC. The two components of the $e$ bending are called the $x$ - and $y$-modes and are shown in the upper row of Figure 1, along with the bending angle $(\theta)$ that is proportional to their distortion coordinates $(x$ and $y$ ). All the other internal coordinates are taken from the optimized $\mathrm{PH}_{3}$ structure, following the vertical ionization picture. The ground ${ }^{2} A_{1}$ state and the degenerate first excited ${ }^{2} E_{x, y}$ states of $\mathrm{PH}_{3}^{+}$are derived from removing an electron from the highest occupied molecular orbital (HOMO) $2 a_{1}$ and the degenerate HOMO-1 $1 e_{x, y}$ of $\mathrm{PH}_{3}$, respectively. The orbitals are shown in the second row of Figure 1 (see Figure S.1 in SI for all seven active orbitals). The vertical ionization energies to the ${ }^{2} A_{1}$ and ${ }^{2} E$ states are calculated to be 10.34 and $13.42 \mathrm{eV}$, only $2 \%$ off from the 10.60 and 13.70 experimental values. ${ }^{89}$ The ${ }^{2} A_{1},{ }^{2} E_{x}$, and ${ }^{2} E_{y}$ states at the $C_{3 v}$ structure are reference prototypes for the MSD diabatization. The obtained diabats are called $Z, X$, and $Y$ correspondingly. The CMOs at distorted structures are rotated to have maximum overlaps with the CMOs at the $C_{3 v}$ structure; the resultant orbitals are the DMOs, and named after their $C_{3 v}$ references.

The non-SOC matrix elements of the three diabats along the bending modes have been thoroughly discussed in Refs. 87 and 88, and they are not the focuses of this work. Here, we only briefly compare our non-SOC matrix elements with those in the two works; a more detailed discussion of the non-SOC matrix elements is given in Section S.III in SI. 
Comparatively, we put more concentration on the SOC matrix elements.

\section{Diabatic non-spin-orbit matrix elements}

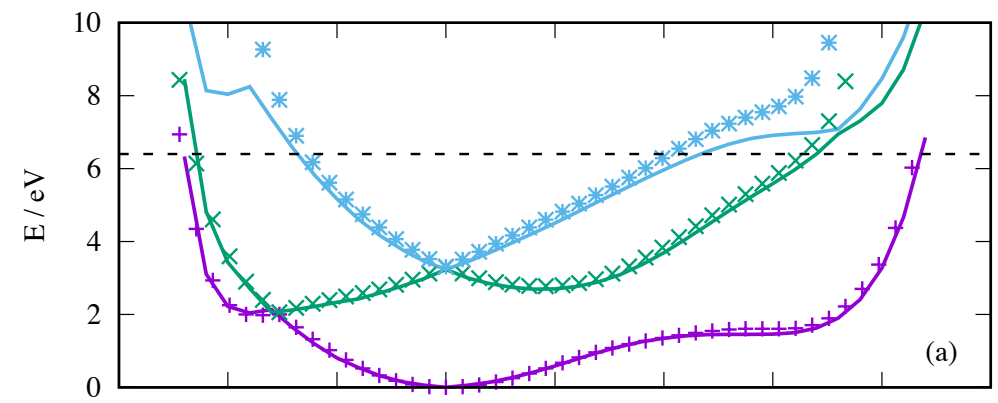

$$
\begin{array}{r}
\mathrm{E}_{1}(\mathrm{TZ})- \\
\mathrm{E}_{2}(\mathrm{TZ})- \\
\mathrm{E}_{3}(\mathrm{TZ})- \\
\mathrm{E}_{1}(\mathrm{BD})+ \\
\mathrm{E}_{2}(\mathrm{BD}) \times \\
\mathrm{E}_{3}(\mathrm{BD}) \quad * \\
\mathrm{E}=6.4 \mathrm{eV}--
\end{array}
$$
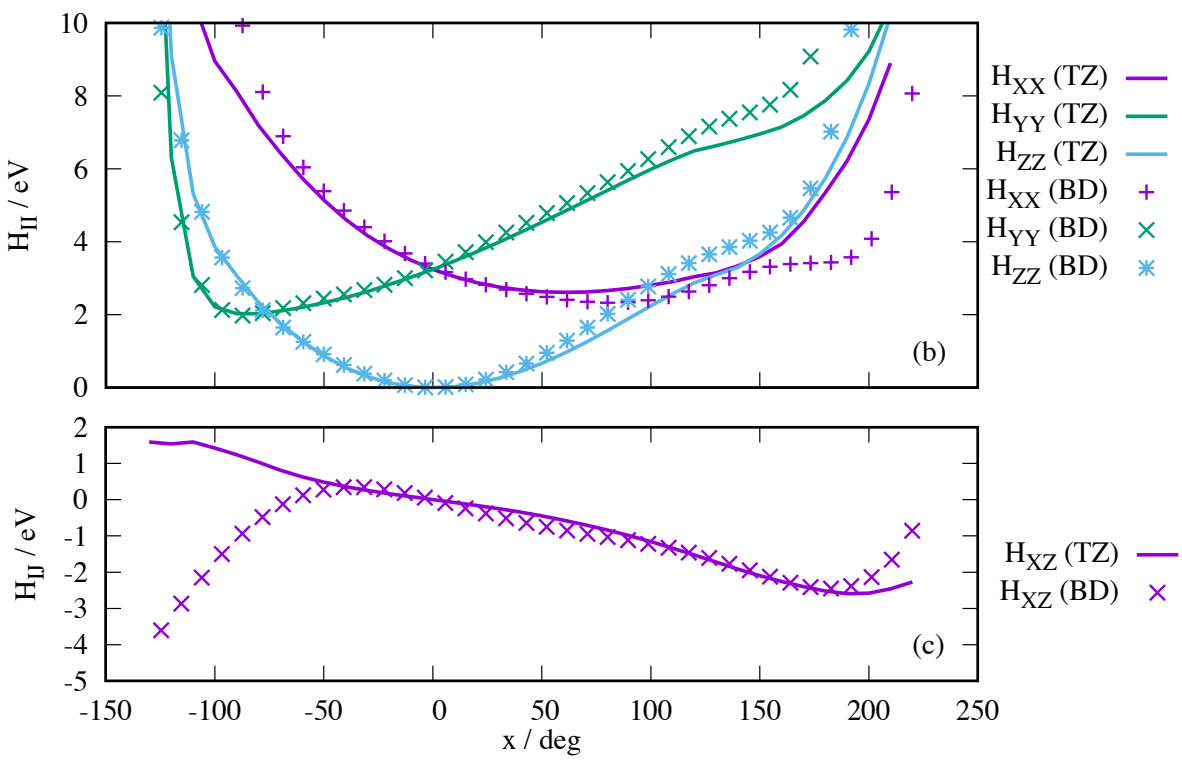

FIG. 2. (a) adiabatic energies, (b) diabatic energies, and (c) diabatic coupling along the $x$-distortion of $\mathrm{PH}_{3}^{+}$. The subscript $i$ for $E_{i}$ in (a) indicates the energy order.

Our calculated non-SOC adiabatic energies and diabatic matrix elements (labelled by "TZ") along the representative $x$-distortion are shown in Figure 2, in comparison with the results reported in Refs. 87 and 88 (labelled by "BD", after the two common authors of the two references). Since the $x$-distortion reduces the symmetry to $C_{s}$, and $|X\rangle$ and $|Z\rangle$ belong to the $A^{\prime}$ irrep while $|Y\rangle$ to $A^{\prime \prime}$, only the $H_{X Z}$ diabatic coupling is nonzero and is shown in Figure 2(c). The energy of $|Z\rangle$ at the undistorted structure is chosen to be the zero reference for the energy curves. The BD adiabatic energies are obtained by diagonalizing the BD diabatic Hamiltonian matrix. The TZ adiabatic energy curves are close to the BD 
ones, especially under $E=6.4 \mathrm{eV}$ (Figure 2(a)). This reflects the similar accuracies of the electronic structure methods, the Multi-Reference Configuration Interaction (MRCI) using the same 7o7e active space in the BD works and the GMC-QDPT method in this work.. Above $E=6.4 \mathrm{eV}$, deviations between the two sets of adiabatic energies become noticeable. This is because the BD diabatization by ansatz only considered adiabatic energies up to the $6.4 \mathrm{eV}$ limit (corresponding to $7 \mathrm{eV}$ if using the BD zero energy reference). Consequently, the BD model cannot reproduce the ab initio calculated adiabatic energies above this limit; it does not capture the crossings and avoided crossings (the breakdown of GBOA) above the limit, e.g, the sharp turn of $E_{3}$ at $x \approx-90^{\circ}$.

Our diabatic elements closely resemble the $\mathrm{BD}$ ones within $-50^{\circ}<x<50^{\circ}$. At larger distortions, along with the differences between the two sets of adiabatic energies, the diabatic matrix elements of the two works differ (Figure 2(b) and (c)). The differences occur far away from the Franck-Condon region and hence the two models should give similar results in simulating the photoelectron spectrum of $\mathrm{PH}_{3}$. For a more detailed discussion of our non-SOC diabatic matrix elements, e.g., the slight discontinuity of $H_{X Z}$ at $x<-120^{\circ}$ in Figure 2(c), and their comparison with the BD results, please see Section S.III in SI.

\section{Diabatic spin-orbit matrix elements}

The two spin components of the spin-doublet diabats are denoted by "+" and "-", for having the $\pm \frac{\hbar}{2}$ spin-projections on the $C_{3}$ axis of the undistorted $\mathrm{PH}_{3}^{+}$. The calculated $\mathrm{SO}$ matrix elements $\left(H_{X+Y+}^{S O}=\left\langle X+\left|\hat{H}_{S O}\right| Y+\right\rangle\right.$, etc. $)$ of $\mathrm{PH}_{3}^{+}$along the $x$ - and $y$-distortions are shown in Figure 3. The trigonal symmetry determines that there are five independent SO matrix elements among the six diabats (doubled from three after considering the spin projections). ${ }^{90}$ Therefore, only the five are shown. Since the SO matrix elements are usually complex, their real and imaginary parts are presented separately.

The SO matrix elements are smooth functions in the wide range of $x$ - and $y$-distortions shown in the figure. The range of distortions shown in the figure has well encompassed the Franck-Condon region of the ionization; the standard deviations of $x$ and $y$ in the ground vibrational state of the neutral $\mathrm{PH}_{3}$ are about $10^{\circ}$ under the harmonic oscillator

approximation. $H_{X+Y+}^{S O}$ and $H_{X+Z-}^{S O}$ are the two nonzero elements among the five at the vertical ionization structure, with magnitudes of $-71 i \mathrm{~cm}^{-1}$ and $-80 \mathrm{~cm}^{-1}$, respectively. 

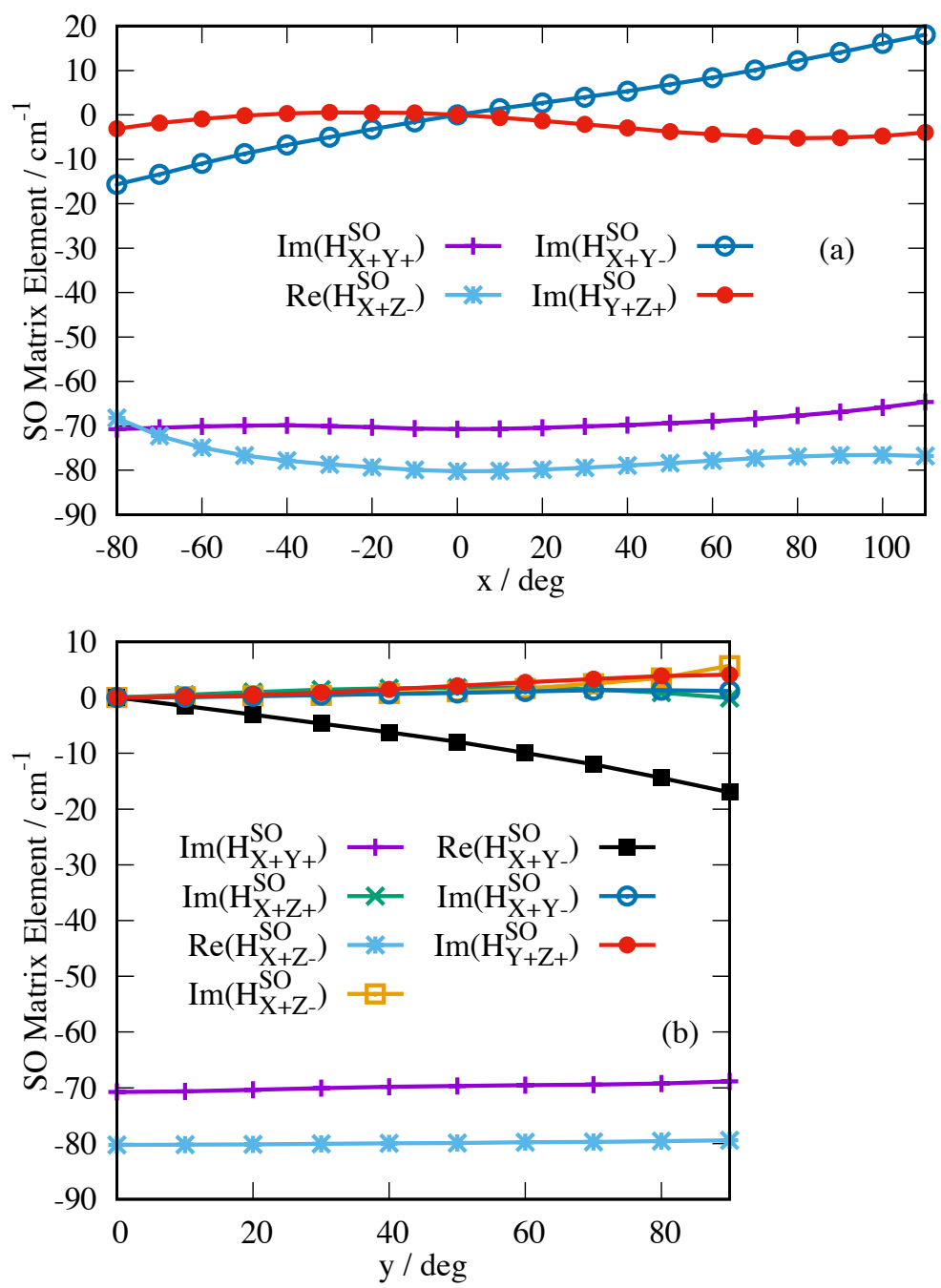

FIG. 3. Calculated $\mathrm{PH}_{3}^{+}$diabatic SO matrix elements along (a) $x$-distortion and (b) $y$-distortion. Only the symmetry-allowed elements are plotted.

They do not vary significantly along the $x$ - and $y$-distortions until $x<60^{\circ}$ for $H_{X+Z-}^{S O}$. The conventional static approximation is valid for those elements. Of more interest to us are the SO elements that gain nonzero values in the distortions. They are responsible for the relativistic Jahn-Teller effect, ${ }^{91}$ and a robust diabatization scheme is needed to describe their geometry-dependence. The main value of the proposed protocol is to calculate the geometry-dependence of diabatic SO elements. The smooth variations of the $\mathrm{SO}$ elements shown in Figure 3 exhibit the reliability of the protocol.

Among the distortion-induced SO elements, $H_{X+Y_{-}}^{S O}$ is the most interesting. It shows the most significant geometry-dependence, and it couples two degenerate diabats at the undistorted structure. Around the origin, it has a $0.16 i \mathrm{~cm}^{-1} /{ }^{\circ}$ slope along the $x$-distortion, 
and $\mathrm{a}-0.16 \mathrm{~cm}^{-1} /{ }^{\circ}$ slope along the $y$-distortion. Assuming that $\mathrm{PH}_{3}$ 's low frequency $e_{x}$ normal mode of $1140 \mathrm{~cm}^{-1}$ contain purely the $x$-bending, the slope is converted to $2.3 i \mathrm{~cm}^{-1}$ with respect to the dimensionless reduced coordinate of the normal mode. This linear SOvibronic coupling constant is negligible when compared to the $1140 \mathrm{~cm}^{-1}$ gap between the vibronic bases that it couples, e.g., $|X+\rangle|0\rangle$ and $|Y-\rangle|1\rangle$. In the second ket of each basis is the vibrational quantum number $v_{x}$ of the $x$-dominated normal mode. The other SO elements vary even less in the FC region. The relativistic JT effect is too small to be observed in the photoelectron spectrum of $\mathrm{PH}_{3}$. Therefore, they are ignored in Bhattacharyya et al.'s calculation of the $\mathrm{PH}_{3}$ photoelectron spectrum. ${ }^{88}$ However, these small SO elements provide very good examples to exhibit the robustness of the protocol: it is accurate enough to capture their subtle geometry-dependence. For systems containing heavier atoms, the protocol should work better.

\section{Origin of the distortion-induced spin-orbit couplings}

An advantage of using the present wave-function-based diabatization scheme is that we can investigate the origin of the distortion-induced SOCs through analyzing the diabats and DMOs. We first examine the most geometry-dependent $H_{X+Y-}^{S O}$. Ignoring the inactive doubly occupied orbitals, $|X+\rangle$ and $|Y-\rangle$ are dominated by the following Slater determinants at the undistorted structure:

$$
\begin{aligned}
& |X+\rangle \approx\left|1 e_{x} \alpha 1 e_{y} \alpha 1 e_{y} \beta 2 a_{1} \alpha 2 a_{1} \beta\right\rangle \\
& |Y-\rangle \approx\left|1 e_{x} \alpha 1 e_{x} \beta 1 e_{y} \beta 2 a_{1} \alpha 2 a_{1} \beta\right\rangle
\end{aligned}
$$

where $\alpha$ and $\beta$ stand for electronic spin functions. According to the Slater-Condone rule, $H_{X+Y}^{S O}$ involves the orbital integral

$$
\left\langle 1 e_{y} \alpha\left|\hat{\vec{z}}_{e f f}^{(1)} \cdot \hat{\vec{s}}\right| 1 e_{x} \beta\right\rangle=\left\langle 1 e_{y}\left|\hat{\vec{z}}_{e f f, x}^{(1)}\right| 1 e_{x}\right\rangle\left\langle\alpha\left|\hat{s}_{x}\right| \beta\right\rangle+\left\langle 1 e_{y}\left|\hat{\vec{z}}_{e f f, y}^{(1)}\right| 1 e_{x}\right\rangle\left\langle\alpha\left|\hat{s}_{y}\right| \beta\right\rangle .
$$

$\left\langle\alpha\left|\hat{s}_{z}\right| \beta\right\rangle=0$ has been used to drop the $\hat{\vec{z}}_{\text {eff,z }}^{(1)} \hat{s}_{z}$ term in the dot product. $\hat{\vec{z}}_{\text {eff }}^{(1)}$ is an effective angular moment operator (see Eqs. S.1 and S.4 in SI) and hence shares the same symmetry with the angular momentum operator $\hat{\vec{l}}$ centered at P. Being the only heavy atom, the central P's $p_{x}$ functions in the $1 e_{x}, p_{y}$ in $1 e_{y}$, and $p_{z}$ in $2 a_{1}$ are responsible for the SO elements of the three DMOs. It is well known that $\left\langle p_{i}\left|\hat{l}_{j}\right| p_{k}\right\rangle \neq 0$ only when the subscripts $i, j$, and $k$ 
follow a cyclic permutation of $x, y$, and $z$, or a pairwise exchange of a cyclic permutation. Therefore, $\left\langle 1 e_{y}\left|\hat{\vec{z}}_{e f f, x}^{(1)}\right| 1 e_{x}\right\rangle=\left\langle 1 e_{y}\left|\hat{\vec{z}}_{e f f, y}^{(1)}\right| 1 e_{x}\right\rangle=0$ in Eq. 5, making $H_{X+Y-}^{S O}=0$ at the undistorted structure.

The $1 e_{x}$ orbital at the undistorted structure is sketched in Figure 4(a). It is mainly a bonding addition of the $3 p_{x}$ orbital of $\mathrm{P}$ and the $\frac{2}{\sqrt{6}} 1 s_{A}-\frac{1}{\sqrt{6}} 1 s_{B}-\frac{1}{\sqrt{6}} 1 s_{C}$ combination of the $\mathrm{H} 1 s$ orbitals. The $1 s$ subscripts $A / B / C$ follow the same labeling in Figure 1 . Along the $x$-distortion, the $1 e_{x}$ DMO has its $1 s_{B}$ and $1 s_{C}$ components follow the scissoring $\mathrm{PH}$ bonds (Figure $4(\mathrm{a})$ ) in order to maintain its character. With a larger $H_{B}$-P- $H_{C}$ bond angle, the $\left\langle 1 s_{B} \mid 3 p_{x}\right\rangle$ and $\left\langle 1 s_{C} \mid 3 p_{x}\right\rangle$ overlaps are smaller. Consequently, after its renormalization, the $1 e_{x}$ DMO contains larger amplitudes of $1 s_{B}$ and $1 s_{C}$. The correlation between the smaller overlaps and the larger atomic orbital amplitudes in a bonding orbital is the best illustrated by the simple expression for the bonding orbital of $\mathrm{H}_{2}$ :

$$
\sigma_{g}=\sqrt{\frac{1}{2\left(1+S_{A B}\right)}}\left(1 s_{A}+1 s_{B}\right),
$$

where $S_{A B}=\left\langle 1 s_{A} \mid 1 s_{B}\right\rangle$. When $S_{A B}$ becomes smaller, the amplitude of each $1 s$ orbital becomes larger. With the larger lobes of $1 s_{B / C}$, The $2:-1:-1$ ratio of $1 s_{A / B / C}$ in $1 e_{x}$ is broken, and the $\left\langle 1 s_{A / B / C} \mid p_{z}\right\rangle$ integrals do not cancel exactly in the distorted $1 e_{x}$ DMO; $\left\langle 1 e_{x} \mid p_{z}\right\rangle \neq 0$. Here $p_{z}$ stands for any $p_{z}$-type basis functions of $\mathrm{P}$. The $1 e_{x} \mathrm{DMO}$ along the $x$ distortion hence must contain some $p_{z}$ character. This argument is quantitatively supported by the purple curve in Figure 4(d). The curve shows the coefficient of the representative basis function that makes the largest contribution among all $p_{z}$ functions to the $1 e_{x}$ DMO; its magnitude increases along with the $x$-distortion. $\left\langle 1 e_{y}\left|\hat{\vec{z}}_{\text {eff } x}^{(1)}\right| p_{z}\right\rangle \neq 0$ (note the yxz permutation) and therefore, $\left\langle 1 e_{y}\left|\hat{\vec{z}}_{e f f, x}^{(1)}\right| 1 e_{x}\right\rangle \neq 0$ when $x$-distorted, making $H_{X+Y-}^{S O} \neq 0$. Since $\left\langle 1 e_{y}\left|\hat{\vec{z}}_{e f f, x}^{(1)}\right| p_{z}\right\rangle$ is imaginary (all angular momentum integrals of real orbitals are imaginary) and the spin factor $\left\langle\alpha\left|\hat{s}_{x}\right| \beta\right\rangle$ is real (recalling the real $\sigma_{x}$ Pauli matrix), $H_{X+Y-}^{S O}$ is purely imaginary along the $x$-distortion (Figure $3(\mathrm{a})$ ).

The $y$-distortion induces nonzero $H_{X+Y}^{S O}$ following a similar mechanism, which is shown in Figure 4(b). The $y$-distortion increases the $\left\langle 1 s_{B} \mid 3 p_{y}\right\rangle$ and reduces the $\left\langle 1 s_{C} \mid 3 p_{y}\right\rangle$ overlap. After renormalization, the distorted $1 e_{y}$ DMO contains a smaller (larger) $1 s_{B}\left(1 s_{C}\right)$ amplidute. The original exact cancelling of the $\left\langle 1 s_{B} \mid p_{z}\right\rangle$ and $\left\langle 1 s_{C} \mid p_{z}\right\rangle$ integrals in $\left\langle 1 e_{y} \mid p_{z}\right\rangle$ is removed, leading to $\left\langle 1 e_{y} \mid p_{z}\right\rangle \neq 0$. The $p_{z}$ admixture in the $1 e_{y}$ DMO is shown by the green curve in Figure $4(\mathrm{~d}) . \quad\left\langle p_{z}\left|\hat{\vec{z}}_{\text {eff,y }}^{(1)}\right| 1 e_{x}\right\rangle \neq 0$ and hence $\left\langle 1 e_{y}\left|\hat{\vec{z}}_{e f f, y}^{(1)}\right| 1 e_{x}\right\rangle \neq 0$ at the 
(a)

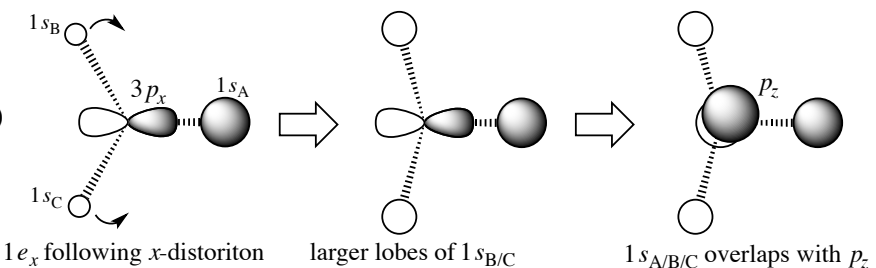

(b)

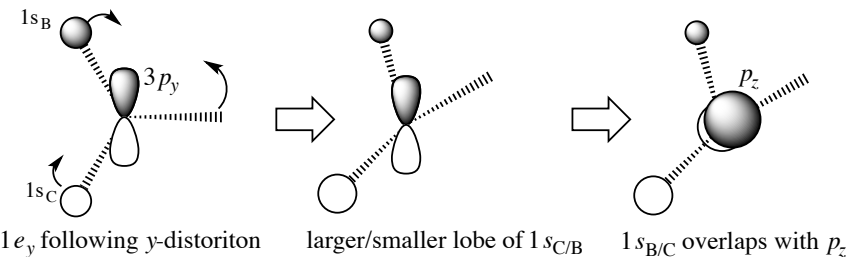

(c)
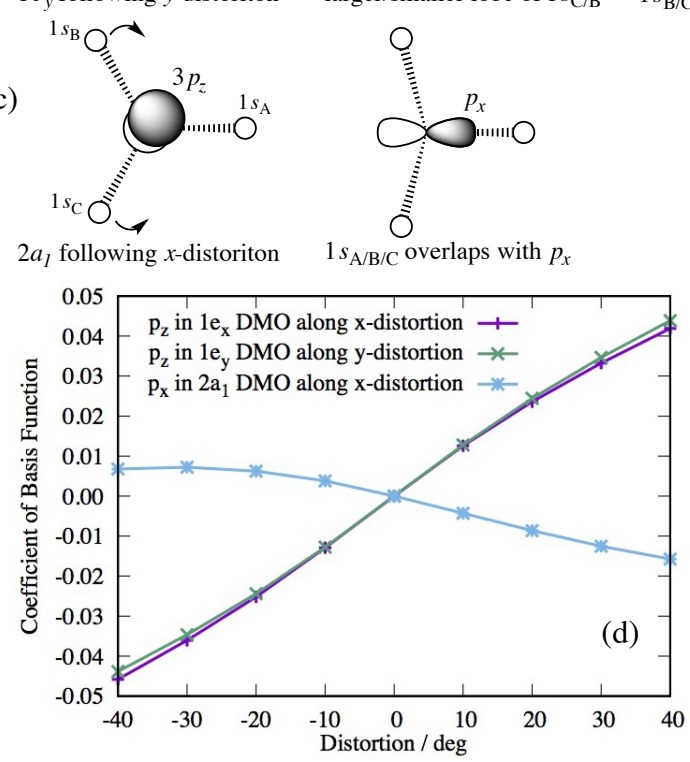

FIG. 4. Variations of (a) the $1 e_{x}$ DMO along the $x$-distortion, (b) the $1 e_{y}$ DMO along the $y$ distortion, and (c) the $2 a_{1} \mathrm{DMO}$ along the $x$-distortion. Plotted in (d) are the coefficients of the mixed-in basis functions in the DMOs along the $x$ - and $y$-distortions. Only coefficients of the $\mathrm{P} p_{x}$ or $p_{z}$ basis functions that make the largest contributions to the respective DMOs are included.

$y$-distortion, making $H_{X+Y-}^{S O} \neq 0$. Both $\left\langle 1 e_{y}\left|\hat{\vec{z}}_{e f f, y}^{(1)}\right| 1 e_{x}\right\rangle$ and $\left\langle\alpha\left|\hat{s}_{y}\right| \beta\right\rangle$ are imaginary (recalling the imaginary $\sigma_{y}$ Pauli matrix), so that their product is real, and so is the $H_{X+Y-}^{S O}$ in Figure 3(b). $\operatorname{Im}\left(H_{X+Y_{-}}^{S O}\right)$ and $\operatorname{Re}\left(H_{X+Y_{-}}^{S O}\right)$ increase in magnitude with identical slopes $\left(0.16 i\right.$ and $\left.-0.16 \mathrm{~cm}^{-1} /{ }^{\circ}\right)$ along the $x$ - and $y$-distortions around the undistorted structure. This is determined by the $C_{3 v}$ symmetry of the problem. ${ }^{90}$ Correspondingly, the $1 e_{x}$ and $1 e_{y}$ DMOs have their $p_{z}$ components increase with similar (identical up to $10^{\circ}$ distoriton) slopes along the respective $x$ - and $y$-distortions (Figure $4(\mathrm{~d})$ ).

The second most geometry-dependent SO element around the undistorted structure is 
$H_{Y+Z+}^{S O}$ along the $x$-distortion. The two diabats are dominated by the two Slater determinants at the undistorted structure:

$$
\begin{aligned}
& |Y+\rangle \approx\left|1 e_{x} \alpha 1 e_{x} \beta 1 e_{y} \alpha 2 a_{1} \alpha 2 a_{1} \beta\right\rangle ; \\
& |Z+\rangle \approx\left|1 e_{x} \alpha 1 e_{x} \beta 1 e_{y} \alpha 1 e_{y} \beta 2 a_{1} \alpha\right\rangle .
\end{aligned}
$$

$H_{Y+Z+}^{S O}$ hence involves $\left\langle 2 a_{1}\left|\hat{z}_{\text {eff,z }}^{(1)}\right| 1 e_{y}\right\rangle\left\langle\beta\left|\hat{s}_{z}\right| \beta\right\rangle$ and the spatial integral $\left\langle 2 a_{1}\left|\hat{z}_{\text {eff,z }}^{(1)}\right| 1 e_{y}\right\rangle=0$ at the undistorted structure. The $2 a_{1}$ orbital is sketched in Figure 4(c), and it mainly contains the $3 p_{z}$ orbital of $\mathrm{P}$ and the $\sqrt{\frac{1}{3}}\left(1 s_{A}+1 s_{B}+1 s_{C}\right)$ combination. The $x$-distortion reduces the $\left\langle 1 s_{B / C} \mid p_{x}\right\rangle$ overlaps; they cannot cancel the $\left\langle 1 s_{A} \mid p_{x}\right\rangle$ integral as in the undistorted structure. Therefore, there is $p_{x}$ admixture in the $2 a_{1}$ DMO along the distortion (see the cyan curve in Figure $4(\mathrm{~d})$ ), making $\left\langle p_{x}\left|\hat{z}_{\text {eff,z }}^{(1)}\right| 1 e_{y}\right\rangle \neq 0$, and hence $\left\langle 2 a_{1}\left|\hat{z}_{\text {eff,z }}^{(1)}\right| 1 e_{y}\right\rangle \neq 0$. Combining the imaginary spatial integral and the real $\left\langle\beta\left|\hat{s}_{z}\right| \beta\right\rangle$ gives the purely imaginary $H_{X+Y_{-}}^{S O}$ in Figure $3(\mathrm{a})$. Note that $H_{X+Z+}^{S O}$ and $H_{Y+Z+}^{S O}$ are connected by symmetry ${ }^{90}$ and the latter has a similar magnitude of geometry-dependence. However, it varies most substantially at the diagonal of the $x-y$ plane (see Figure 6 (c) below) and therefore is not outstanding in Figure 3.

The smaller amount of the $p_{x}$ contaminant in the $2 a_{1}$ DMO than the $p_{z}$ in the $1 e_{x}$ DMO (the cyan vs. the purple curve in Figure $4(\mathrm{~d})$ ) is attributed to the smaller magnitudes of $1 s_{B}$ and $1 s_{C}$ in $2 a_{1}:\left\langle 1 s_{B / C} \mid 2 a_{1}\right\rangle=0.200$ vs. $\left\langle 1 s_{B / C} \mid 1 e_{x}\right\rangle=0.326$ at the undistorted structure. With the smaller magnitudes, they are less capable of mediating the $p_{x}$ admixture into the $2 a_{1}$ DMO than mediating $p_{z}$ into the $1 e_{x}$ DMO when $x$-distorted, and give the smaller magnitude of the distortion-induced $H_{Y+Z+}^{S O}$ than $H_{X+Y-}^{S O}$.

SOC is largely an effective 1-electron interaction. Therefore, it is natural to use orbitals to describe this interaction and its consequences. ${ }^{92}$ In the orbital language, the origin of the distortion-induced SOCs, i.e., the relativistic JT effect, is attributed to the symmetrylowering of the diabatic molecular orbitals and the consequently activated spatial angular momentum integrals.

\section{B. Arsine and stibine cations}

As the central atom becomes heavier, $\mathrm{AsH}_{3}^{+}$and $\mathrm{SbH}_{3}^{+}$feature stronger $\mathrm{SO}$ interaction than $\mathrm{PH}_{3}^{+}$. The calculated $\mathrm{SO}$ matrix elements of the two cations are shown in Figure 5 . 
The origins correspond to the optimized structures of the respective neutral hydrides. Only the $x$-distortion is considered because, as mentioned above, $H_{X+Y-}^{S O}$ has a similar rising slope along the $y$-distortion due to symmetry. And this most geometry-dependent element serves the best to demonstrate the robustness of the present protocol.

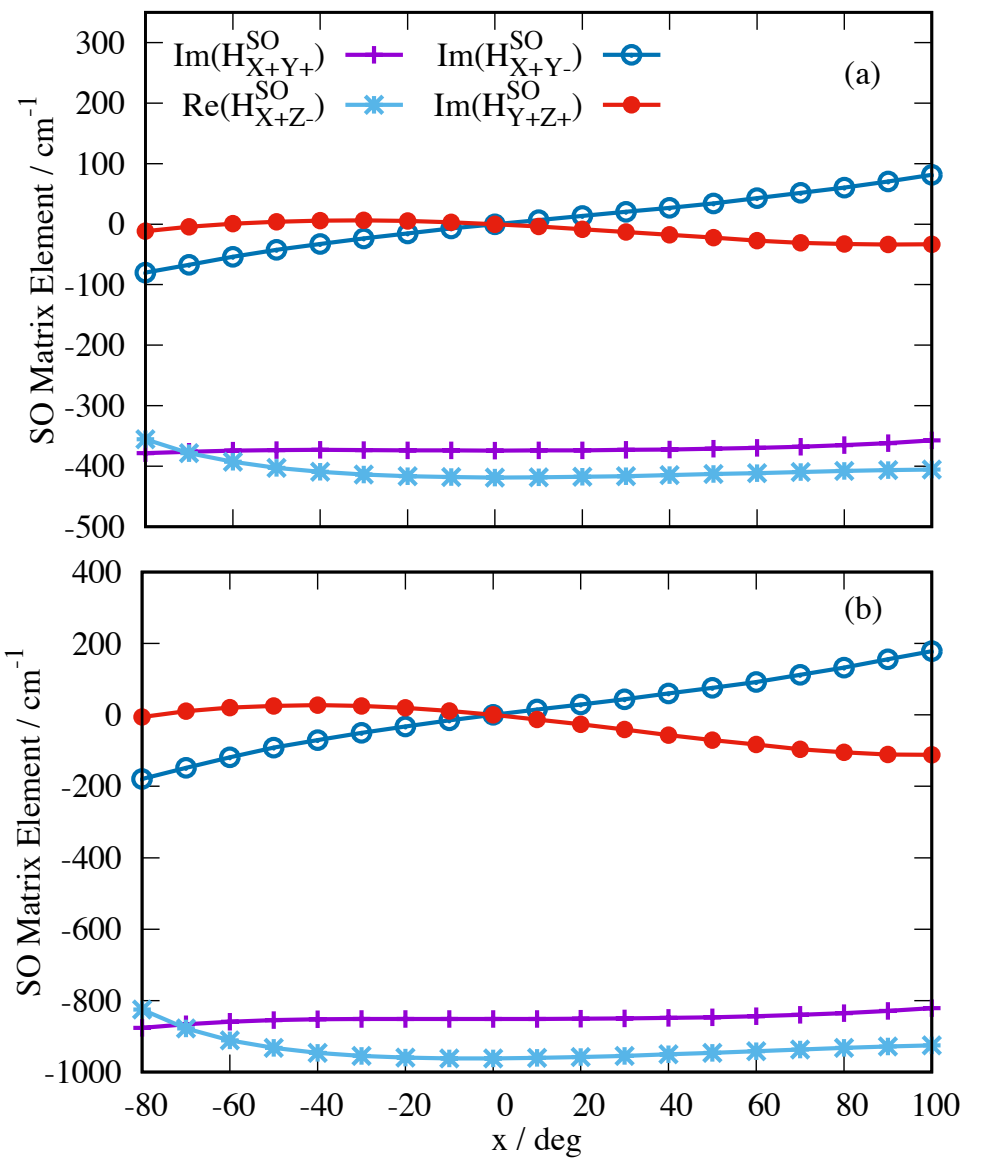

FIG. 5. Calculated (a) $\mathrm{AsH}_{3}^{+}$and (b) $\mathrm{SbH}_{3}^{+}$diabatic $\mathrm{SO}$ matrix elements along the $x$-distortion. Only the symmetry-allowed elements are plotted.

The SO elements increase significantly in magnitude, with, e.g., $H_{X+Y+}^{S O}=-374 i$ and $-851 i \mathrm{~cm}^{-1}$ and $H_{X+Z_{-}}^{S O}=-419$ and $-962 \mathrm{~cm}^{-1}$ for $\mathrm{AsH}_{3}^{+}$and $\mathrm{SbH}_{3}^{+}$at their undistorted structures, compared to the $-71 i \mathrm{~cm}^{-1}$ and $-80 \mathrm{~cm}^{-1}$ counterparts of $\mathrm{PH}_{3}^{+}$. These SO elements of $\mathrm{AsH}_{3}^{+}$and $\mathrm{SbH}_{3}^{+}$can be approximated by scaling the $\mathrm{PH}_{3}^{+}$counterparts by 5.3 and 12. The variations of the SO elements along the $x$-distortion are also more significant than in $\mathrm{PH}_{3}^{+}$. The profile of the geometry-dependences of the $\mathrm{SO}$ elements is similar to those of $\mathrm{PH}_{3}^{+}$. If the elements in Figure 3(a) are scaled by 5.3 and 12, the results are close to those in Figure 5 (see Figure S.6 in SI). This is reasonable since the similar mechanism of 
orbital mixing underlies those distortion-induced SOCs. In addition to the heavier pnicogen atoms, the larger $1 s$ amplitudes in the DMOs, e.g., $\left\langle 1 s_{A / B / C} \mid 2 a_{1}\right\rangle=0.256$ in $\mathrm{SbH}_{3}^{+}$vs. 0.200 in $\mathrm{PH}_{3}^{+}$, also contribute to the more substantial geometry-dependence of the $\mathrm{SO}$ elements. The larger $1 s$ amplitudes result from the increased electropositivity of the heavy pnicogen atoms. They are more capable of mediating the orbital mixings shown in Figure 4 and hence increase the distortion-induced SOCs.

\section{Fitting}

The point by point calculated SO elements can be fitted using symmetry-adapted multidimensional polynomials (the fitting of the non-SOC matrix elements is discussed in Section S.V in SI). Some matrix elements, e.g., $H_{X+Z+}^{S O}$ and $H_{Y+Z+}^{S O}$, are related by symmetry and share a common set of coefficients in their polynomial expansions; their fitting requires a nonlinear algorithm. The Marquardt-Levenberg algorithm ${ }^{93,94}$ is used to fit the parametershared polynomials together. For instance, the following function,

$\chi^{2}=\sum_{i}\left[\left(P_{X+Z+}\left(x_{i}, y_{i}, z_{i}\right)-H_{X+Z+}^{S O}\left(x_{i}, y_{i}, z_{i}\right)\right)^{2}+\left(P_{Y+Z+}\left(x_{i}, y_{i}, z_{i}\right)-H_{Y+Z+}^{S O}\left(x_{i}, y_{i}, z_{i}\right)\right)^{2}\right]$

is minimized to get the common set of parameters for $H_{X+Z+}^{S O}$ and $H_{Y+Z+}^{S O} \cdot\{i\}$ index the sampled distortions for which the $H_{I J}^{S O}$ elements are calculated, and $P_{I J}$ are the polynomials to be fitted. In sampling the distortions, we set $\theta_{x y}=\operatorname{atan}(y / x)$ and $r_{x y}= \pm \sqrt{x^{2}+y^{2}}$, and sample the $x$ - $y$-distortions from $\theta=0$ to $90^{\circ}$, with a $15^{\circ}$ step size; at each $\theta, r_{x y}$ is varied by a $10^{\circ}$ step size in both positive and negative directions. Since all matrix elements are either symmetric or antisymmetric with respect to the $y$-distortion, sampling the first and third quadrants of the $x-y$ plane in this way is enough.

The SO matrix elements are fitted using the 4-th order polynomials given in Appendix A of Ref. 90. The fitted polynomials of the representative, most geometry-dependent $H_{X+Y-}^{S O}$, $H_{X+Z+}^{S O}$ and $H_{Y+Z+}^{S O}$ of $\mathrm{PH}_{3}^{+}$are plotted and compared with the point-by-point calculated values in Figure 6. The advantage using the polar coordinates in sampling the $x$ - $y$-distortions is clearly shown in the figure: a higher density of configurations around the FC region are sampled. The calculated references are all close to the fitted surfaces, with the small $\sim 0.3 \mathrm{~cm}^{-1}$ averaged fitting errors for all four panels (please also see Figure S.8 in SI for 

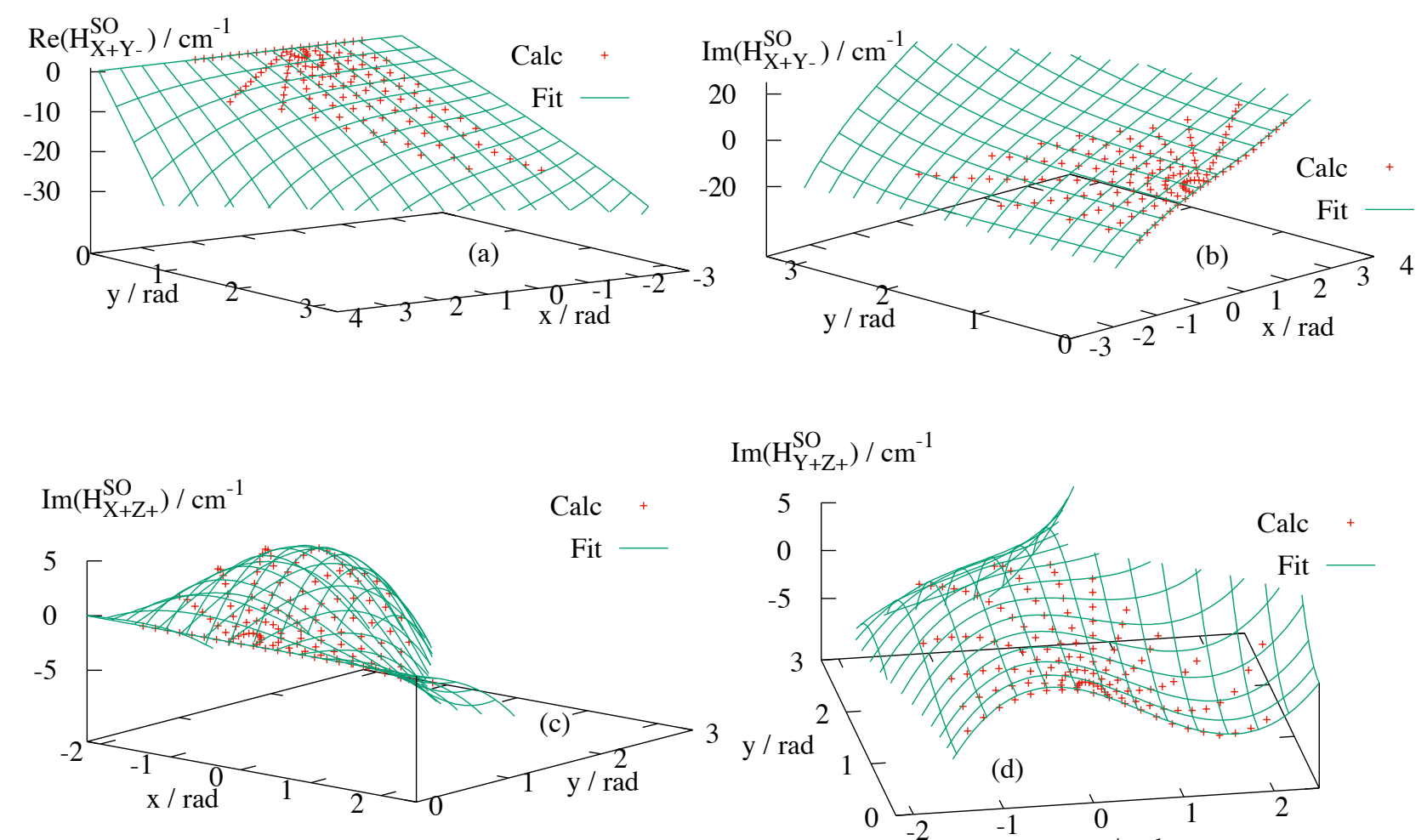

$$
\operatorname{Im}\left(\mathrm{H}_{\mathrm{Y}+\mathrm{Z}+}^{\mathrm{SO}}\right) / \mathrm{cm}^{-1}
$$

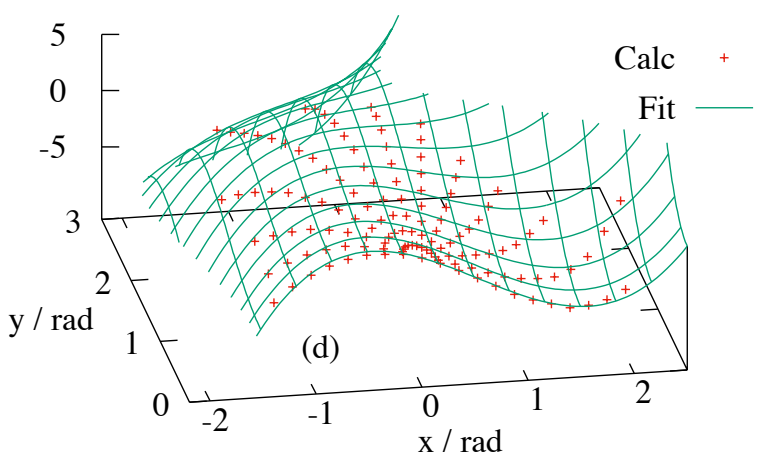

FIG. 6. The calculated and fitted representative diabatic $\mathrm{SO}$ matrix elements of $\mathrm{PH}_{3}^{+}$: (a) $\operatorname{Re}\left(H_{X+Y_{-}}^{S O}\right)$, (b) $\operatorname{Im}\left(H_{X+Y-}^{S O}\right),(\mathrm{c}) \operatorname{Im}\left(H_{X+Z+}^{S O}\right)$, and (d) $\operatorname{Im}\left(H_{Y+Z+}^{S O}\right)$.

the fitting residuals, most of which are $\left.<0.5 \mathrm{~cm}^{-1}\right)$. The fittings of other $H_{I J}^{S O}$ have similar small errors. The curvy $\operatorname{Im}\left(H_{X+Z+}^{S O}\right)$ and $\operatorname{Im}\left(H_{Y+Z+}^{S O}\right)$ surfaces in Figure 6(c) and (d) exhibit the need of using the 4 -th order polynomials to represent the SO elements.

In terms of fitting, the present protocol features the following advantages in comparison with the diabatization by ansatz. First, the diabatic matrix elements are fitted directly, instead of through the eigenvalues of the Hamiltonian matrix. Matrix diagonalization is not needed in the fitting, and the analytical gradients of $\chi^{2}$ with respect to the fitting parameters are known. This enhances the stability and facilitates the convergence of the fitting, and makes it less dependent on the initial parameters. ${ }^{30}$ Second, the fitting function of the present protocol (e.g., Eq. 9) only contains the SO elements that share a common set of parameters, while the fitting function in the diabatization by ansatz contains all elements, SO and nonSO. The fitting in the present protocol is intrinsically simpler. Third, the fittings of the non-SO and SO elements are entangled in the diabatization by ansatz. The fitting error of the former is inevitably transferred to the latter, despite the large difference in their absolute 
values (the non-SO elements are usually given in the unit of eV, while the $\mathrm{SO}$ elements in $\mathrm{cm}^{-1}$ ). The typical hundreds of $\mathrm{cm}^{-1}$ fitting error of the diabatization by ansatz may just be a small fraction of the non-SO elements, but can be larger than the absolute values of the SO elements. In the present protocol, the SO elements are calculated and fitted separately, with the commensurate, small fitting errors $\left(\sim 0.3 \mathrm{~cm}^{-1}\right)$. We have no intention to claim that the present protocol is superior to the diabatization by ansatz, especially considering the convenience of the latter when wave function information is unavailable. The present protocol is a supplement for the other diabatization schemes.

\section{CONCLUSIONS}

We present a diabatization protocol that can estimate the spin-orbit couplings between diabatic states accurately. The adiabat-to-diabat transformation matrix is prepared using the recently developed model space diabatization scheme. The scheme is adapted so that it can handle wave functions with more flexible active spaces, and it is easy to keep the phases of the diabats consistent. The spin-orbit couplings between adiabatic states are then transformed to the couplings between diabatic states using the matrix. The spin-orbit coupling calculation involves a mean-field treatment for the two-electron interaction. To test its robustness, the protocol is employed to prepare the three diabatic states that are the most relevant for the low energy photoelectron spectra of phosphine, arsine, and stibine, and calculate the spin-orbit couplings among them. The central results of this work are the geometry-dependences of the diabatic spin-orbit matrix elements shown in Figures 3, 5, and 6. They vary up to hundreds of $\mathrm{cm}^{-1}$ along the degenerate bending distortion. This magnitude of variation is within the typical $\sim 400 \mathrm{~cm}^{-1}$ fitting error of the diabatization by ansatz; it is difficult to use this conventional method to describe these spin-orbit couplings. Calculating the spin-orbit couplings directly, the present protocol encounters no difficulty in getting their values and geometry-dependences. The new protocol serves as a supplement for the other diabatization schemes when an accurate description of spin-orbit coupling for a wide range of distortion is needed.

The protocol features the following additional advantages. (1) Its efficiency is promising. Maximally $20 \%$ additional compute time on top of the underlying ab initio calculation is needed. (2) As the diabatization and spin-orbit calculation are carried out on the fly, the $a$ 
priori knowledge of asymptotic atomic spin-orbit couplings is not needed. (3) With the wave functions of the diabatic states available, it is straightforward to investigate the origin of the distortion-induced spin-orbit couplings. For the pnicogen hydride cations considered, all the distortion-induced couplings arise from the orbital mixings in symmetry-lowering, which make the angular momentum integrals of the relevant orbitals nonzero. (4) The direct fitting of the diabatic matrix elements without diagonalizing the matrix is efficient and numerically stable. With all these advantages, we foresee a wide use of the protocol in the future.

\section{SUPPLEMENTARY MATERIALS}

See supplementary material for the following contents: the mean-field treatment of twoelectron spin-orbit interaction; the plotting of all 7 active orbitals of $\mathrm{PH}_{3}^{+}$; non-spin-orbit diabatic matrix elements of $\mathrm{PH}_{3}^{+}$along the e-bending; comparison of the scaled spin-orbit matrix elements of $\mathrm{PH}_{3}^{+}$and the calculated elements of $\mathrm{AsH}_{3}^{+}$and $\mathrm{SbH}_{3}^{+}$; fitting of the nonspin-orbit matrix elements; fitting errors of the representative spin-orbit matrix elements.

\section{ACKNOWLEDGEMENTS}

Computations were made on the supercomputer Mammoth parallelèl 2 from Université de Sherbrooke, managed by Calcul Québec and Compute Canada, and on the General Purpose Cluster at the SciNet HPC Consortium, also managed by Compute Canada. T.Z. is grateful to Wolfgang Domcke (Technische Universität München) and Swarnendu Bhattacharyya (École Polytechnique Fédérale de Lausanne) for enlightening discussion and sharing their $\mathrm{PH}_{3}^{+}$diabatic potential and coupling surfaces. T.Z. thanks Mark Gordon (Iowa State University) and Mike Schmidt (Iowa State University) for their continuous development of the GAMESS-US program package. T.Z. is thankful to the Natural Sciences and Engineering Research Council (NSERC) of Canada for research funding (RGPIN-2016-06276), and Carleton University for start-up grant (186853). 
1 L. S. Cederbaum, in Conical intersections: electronic structure, dynamics and spectroscopy, edited by W. Domcke, D. R. Yarkony, and H. Köppel (World Scientific, 2004) Chap. 1, pp. $3-40$.

2 D. R. Yarkony, Chem. Rev. 112, 481 (2011).

3 J. C. Tully, J. Chem. Phys. 137, 22A301 (2012).

4 B. C. Garrett and D. G. Truhlar, in Theoretical Chemistry: Advances and Perspectives, Vol. 6A, edited by D. Henderson (Academic Press, Inc., New York, 1981) pp. 215-289.

5 B. Andresen and S. E. Nielsen, Mol. Phys. 21, 523 (1971).

6 C. A. Mead and D. G. Truhlar, J. Chem. Phys. 77, 6090 (1982).

7 H. Köppel, W. Domcke, and L. S. Cederbaum, Adv. Chem. Phys. 59, 57 (1984).

8 T. Pacher, L. S. Cederbaum, and H. Köppel, Adv. Chem. Phys. 84, 293 (1993).

9 B. K. Kendrick, C. A. Mead, and D. G. Truhlar, Chem. Phys. 277, 31 (2002).

10 J. E. Subotnik, S. Yeganeh, R. J. Cave, and M. A. Ratner, J. Chem. Phys. 129, 244101 (2008).

11 X. Zhu and D. R. Yarkony, J. Phys. Chem. A 119, 12383 (2015).

12 H.-J. Werner and W. Meyer, J. Chem. Phys. 74, 5802 (1981).

13 R. J. Cave and J. F. Stanton, J. Chem. Phys. 144, 054110 (2016).

14 C. E. Hoyer, X. Xu, D. Ma, L. Gagliardi, and D. G. Truhlar, J. Chem. Phys. 141, 114104 (2014).

15 C. E. Hoyer, K. Parker, L. Gagliardi, and D. G. Truhlar, J. Chem. Phys. 144, 194101 (2016).

16 A. Thiel and H. Köppel, J. Chem. Phys. 110, 9371 (1999).

17 C. Woywod, W. Domcke, A. L. Sobolewski, and H.-J. Werner, J. Chem. Phys. 100, 1400 (1994).

18 H. Köppel, J. Gronki, and S. Mahapatra, J. Chem. Phys. 115, 2377 (2001).

19 T. Ichino, J. Gauss, and J. F. Stanton, J. Chem. Phys. 130, 174105 (2009).

20 M. Nooijen, Int. J. Quantum Chem. 95, 768 (2003).

21 T. Pacher, L. S. Cederbaum, and H. Köppel, J. Chem. Phys. 89, 7367 (1988).

22 W. Domcke and C. Woywod, Chem. Phys. Lett. 216, 362 (1993).

23 G. J. Atchity and K. Ruedenberg, Theor. Chem. Acc. 97, 47 (1997).

24 K. Ruedenberg and G. J. Atchity, J. Chem. Phys. 99, 3799 (1993).

25 H. Nakamura and D. G. Truhlar, J. Chem. Phys. 115, 10353 (2001). 
26 H. Nakahara and D. G. Truhlar, J. Chem. Phys. 117, 5576 (2002).

27 K. R. Yang, X. Xu, and D. G. Truhlar, Chem. Phys. Lett. 573, 84 (2013).

28 S. L. Li, D. G. Truhlar, M. W. Schmidt, and M. S. Gordon, J. Chem. Phys. 142, 064106 (2015).

29 R. J. Cave and J. F. Stanton, J. Chem. Phys. 140, 214112 (2014).

30 F. Venghaus and W. Eisfeld, J. Chem. Phys. 144, 114110 (2016).

31 N. Wittenbrink, F. Venghaus, D. Williams, and W. Eisfeld, J. Chem. Phys. 145, 184108 (2016).

32 C. M. Marian, "Fine and hyperfine structure: Spin properties of molecules," in Problem Solving in Computational Molecular Science: Molecules in Different Environments, edited by S. Wilson and G. H. F. Diercksen (Kluwer Academic Publishers, Dordrecht, Netherlands, 1997) pp. 291-351.

33 C. M. Marian, "Spin-orbit coupling in molecules," in Reviews in Computational Chemistry, Vol. 17, edited by K. B. Lipkowitz and D. B. Boyd (WILEY-VCH, New York, 2001) pp. 99-204.

34 D. G. Fedorov, S. Koseki, M. W. Schmidt, and M. S. Gordon, Int. Rev. Phys. Chem. 22, 551 (2003).

35 W. Liu, Mol. Phys. 108, 1679 (2010).

36 D. G. Fedorov, M. W. Schmidt, S. Koseki, and M. S. Gordon, "Spin-orbit coupling methods and applications to chemistry," in Recent Advances in Relativistic Molecular Theory, edited by K. Hirao and Y. Ishikawa (World Scientific, Singapore, 2004) pp. 107-136.

37 K. S. Pitzer, Acc. Chem. Res. 12, 271 (1979).

38 P. Pyykkö, Chem. Rev. 88, 563 (1988).

39 T. Zeng, D. G. Fedorov, and M. Klobukowski, J. Chem. Phys. 134, 024108 (2011).

40 J. Tatchen, N. Gilka, and C. M. Marian, Phys. Chem. Chem. Phys. 9, 5209 (2007).

41 J. S. Cohen and B. Schneider, J. Chem. Phys. 61, 3230 (1974).

42 F. Rebentrost and W. A. Lester Jr., J. Chem. Phys. 64, 3879 (1976).

43 R. Valero and D. G. Truhlar, J. Phys. chem. A 111, 8536 (2007).

44 I. B. Bersuker, Chem. Rev. 101, 1067 (2001).

45 B. E. Applegate, T. A. Barckholtz, and T. A. Miller, Chem. Soc. Rev. 32, 38 (2003).

46 W. Domcke, S. Mishra, and L. V. Poluyanov, Chem. Phys. 322, 405 (2006).

47 L. V. Poluyanov and W. Domcke, J. Chem. Phys. 129, 224102 (2008).

48 L. V. Poluyanov and W. Domcke, Chem. Phys. 374, 86 (2010).

49 L. V. Poluyanov and W. Domcke, Chem. Phys. 407, 1 (2012). 
50 L. V. Poluyanov and W. Domcke, J. Chem. Phys. 137, 114101 (2012).

51 Y. Amatatsu, K. Morokuma, and S. Yabushita, J. Chem. Phys. 94, 4858 (1991).

52 Y. Amatatsu, S. Yabushita, and K. Morokuma, J. Chem. Phys. 104, 9783 (1996).

53 D. Opalka, L. V. Poluyanov, and W. Domcke, J. Chem. Phys. 135, 104108 (2011).

54 H. Ndome, R. Welsch, and W. Eisfeld, J. Chem. Phys. 136, 034103 (2012).

55 H. Ndome and W. Eisfeld, J. Chem. Phys. 137, 064101 (2012).

56 N. Wittenbrink, H. Ndome, and W. Eisfeld, J. Phys. Chem. A 117, 7408 (2013).

57 M. H. Alexander, D. E. Manolopoulos, and H.-J. Werner, J. Chem. Phys. 113, 11084 (2000).

58 M. R. Hoffmann and G. C. Schatz, J. Chem. Phys. 113, 9456 (2000).

59 H. Nakano, R. Uchiyama, and K. Hirao, J. Comput. Chem. 23, 1166 (2002).

60 M. Miyajima, Y. Watanabe, and H. Nakano, J. Chem. Phys. 124, 044101 (2006).

61 R. Ebisuzaki, Y. Watanabe, and H. Nakano, Chem. Phys. Lett. 442, 164 (2007).

62 J. Ivanic, J. Chem. Phys. 119, 9364 (2003).

63 G. J. Atchity and K. Ruedenberg, J. Chem. Phys. 111, 2910 (1999).

64 T. Zeng and P. Goel, J. Phys. Chem. Lett. 7, 1351 (2016).

65 T. Zeng, J. Phys. Chem. Lett. 7, 4405 (2016).

66 B. A. Hess, C. M. Marian, U. Wahlgren, and O. Gropen, Chem. Phys. Lett. 251, 365 (1996).

67 A. A. Granovsky, J. Chem. Phys. 134, 214113 (2011).

68 Y.-K. Choe, H. A. Witek, J. P. Finley, and K. Hirao, J. Chem. Phys. 114, 3913 (2001).

69 H. A. Witek, Y.-K. Choe, J. P. Finley, and K. Hirao, J. Comput. Chem. 23, 957 (2002).

70 A. D. Becke, J. Chem. Phys. 96, 2155 (1992).

71 A. D. Becke, J. Chem. Phys. 97, 9173 (1992).

72 A. D. Becke, J. Chem. Phys. 98, 5648 (1993).

73 C. Lee, W. Yang, and R. G. Parr, Phys. Rev. B 37, 785 (1988).

74 T. H. Dunning, J. Chem. Phys. 90, 1007 (1989).

75 D. E. Woon and T. H. Dunning, J. Chem. Phys. 98, 1358 (1993).

76 A. K. Wilson, D. E. Woon, K. A. Peterson, and T. H. Dunning, J. Chem. Phys. 110, 7667 (1999).

77 T. Noro, M. Sekiya, and T. Koga, Theor. Chem. Acc. 131, 1124 (2012).

78 B. A. Hess, Phys. Rev. A 33, 3742 (1986).

79 G. Jansen and B. A. Hess, Phys. Rev. A 39, 6016 (1989). 
80 T. Nakajima and K. Hirao, Chem. Phys. Lett. 329, 511 (2000).

81 T. Nakajima and K. Hirao, J. Chem. Phys. 113, 7786 (2000).

82 M. W. Schmidt, K. K. Baldridge, J. A. Boatz, S. T. Elbert, M. S. Gordon, J. H. Jensen, S. Koseki, N. Matsunaga, K. A. Nguyen, S. Su, T. L. Windus, M. Dupuis, and J. A. Montgomery Jr., J. Comput. Chem. 14, 1347 (1993).

83 M. S. Gordon and M. W. Schmidt, "Advances in electronic structure theory: Gamess a decade later," in Theory and Applications of Computational Chemistry: The First Forty Years, edited by C. E. Dykstra, G. Frenking, K. S. Kim, and G. E. Scuseria (Elsevier, Amsterdam, 2005).

84 I. B. Bersuker and V. Z. Polinger, Vibronic Interactions in Molecules and Crystals (SpringerVerlag, 1989).

85 A. Viel, W. Eisfeld, S. Neumann, W. Domcke, and U. Manthe, J. Chem. Phys. 124, 214306 (2006).

86 S. Bhattacharyya, D. Opalka, L. V. Poluyanov, and W. Domcke, J. Phys. Conf. Series 428, 1 (2013).

87 S. Bhattacharyya, D. Opalka, L. V. Poluyanov, and W. Domcke, J. Phys. Chem. A 118, 11962 (2014).

88 S. Bhattacharyya, Z. Dai, and W. Domcke, J. Chem. Phys. 143, 194301 (2015).

89 R. Maripuu, I. Reineck, H. Ågren, N.-Z. Wu, J. M. Rong, H. Veenhuizen, and S. H. Al-Shamma, Mol. Phys. 48, 1255 (1983).

90 T. Weike and W. Eisfeld, J. Chem. Phys. 144, 104108 (2016).

91 W. Domcke, D. Opalka, and L. V. Poluyanov, J. Chem. Phys. 144, 124101 (2016).

92 T. Zeng, D. G. Fedorov, M. W. Schmidt, and M. Klobukowski, J. Chem. Theory Comput. 7, $2864(2011)$.

93 K. Levenberg, Quart. Appl. Math. 2, 164 (1944).

94 D. W. Marquardt, SIAM J. Appl. Math. 11, 431 (1963). 